Interação em Psicologia, 2007, 11(1), p. 71-80

\title{
O paradigma de priming semântico na investigação do processamento de leitura de palavras
}

\author{
Jerusa Fumagalli de Salles \\ Graciela Inchausti de Jou \\ Universidade Federal do Rio Grande do Sul \\ Lílian Milnitsky Stein \\ Pontifícia Universidade Católica do Rio Grande do Sul
}

\begin{abstract}
RESUMO
O priming é um tipo de memória implícita (não declarativa) referente aos efeitos facilitadores de eventos antecedentes (primes) sobre o desempenho subseqüente (alvos). No priming semântico há uma relação de significado ou de contexto entre o prime e o alvo. Este paradigma pode ser utilizado como recurso metodológico para estudar, de forma indireta, o processamento semântico na leitura. Este artigo visa apresentar conceitos e discutir aspectos metodológicos do paradigma de priming semântico e as possibilidades de uso para estudo do processamento da leitura. Será apresentada uma revisão do conceito e de procedimentos metodológicos do priming semântico nos estudos de leitura. Serão abordados os modelos teóricos de leitura de palavras (acesso ao léxico mental), os modelos explicativos para o efeito de priming semântico na leitura e serão analisadas pesquisas utilizando o paradigma para investigar o reconhecimento de palavras escritas. Conclui-se que, apesar dos estudos internacionais, especialmente os realizados com crianças, conduzirem a resultados não consensuais, o paradigma de priming semântico mostra-se útil para investigar o conhecimento semântico implícito e on-line, ou seja, durante o processamento das palavras lidas, o que torna sua testagem ecológica e menos artificial. As diferenças de resultados podem relacionar-se à variabilidade dos procedimentos metodológicos.
\end{abstract}

Palavras-chave: priming semântico; leitura de palavras; memória implícita.

\begin{abstract}
The semantic priming paradigm in the word reading processing investigation

Priming is a type of implicit memory (non-declarative) referring to the facilitating effects of precedent events (primes) on subsequent performance (targets). In semantic priming there is a relation of signification or context between the prime and the target. This paradigm can be used as a methodological resource to study, in an indirect manner, the semantic processing in reading. This article aims to present concepts and discuss methodological aspects of the paradigm of semantic priming and the possibilities of using it in studying the process of reading. A revision of concepts and the discussion of methodological aspects of the semantic priming paradigm in reading studies will be presented. Theoretical models of word reading (access to mental lexicon), the explanatory models for the semantic priming effect in reading will be approached. And, studies using the paradigm to investigate the recognition of written words will be analyzed. Despite international studies that led to non-consensual results, especially those undertaken with children, this study infers that the semantic priming paradigm shows to be useful in investigating implicit and on-line semantic knowledge, rather, during the process of reading words, which makes its testing more ecological and less artificial. The differences of results can be related to the variability of methodological procedures.
\end{abstract}

Keywords: semantic priming; word reading; implicit memory.

Priming é um tipo de memória implícita (não declarativa) referente aos efeitos facilitadores de eventos antecedentes (primes) sobre o desempenho subseqüente (respostas aos alvos), ou seja, um aperfeiçoa- mento da capacidade de detectar ou identificar palavras, objetos ou figuras após uma experiência recente com eles (Squire \& Kandel, 2003). O paradigma de priming, apesar de ser um recurso metodológico ainda pouco utilizado no Brasil para investigar os processos 
de leitura, permite estudar a relação entre memória implícita e linguagem escrita. O efeito de priming em tarefas lingüísticas (como leitura de palavras), se manifesta quando o processamento de uma palavra (o alvo) é facilitado pelo estímulo antecedente (o prime). A interpretação mais comum do priming é de que a ativação da representação do prime automaticamente ativa a representação do alvo (Forster, 1999).

Os pesquisadores podem estar interessados no estudo do fenômeno de priming per se, em geral comparando os resultados em tarefas de memória explícita e implícita, como a pesquisa de Oliveira e Janczura (2004) e a de Pompéia \& Bueno (2006), ou utilizar o fenômeno de priming como um recurso metodológico para evidenciar outros tipos de processos cognitivos, como a leitura de palavras.

Este artigo tem como objetivos apresentar conceitos e discutir aspectos metodológicos do paradigma de priming semântico e as possibilidades de uso para estudo do processamento da leitura. Inicialmente será apresentada uma breve revisão do conceito e de procedimentos metodológicos do priming semântico nos estudos de leitura. Depois serão abordados os modelos teóricos de leitura de palavras (acesso ao léxico mental) e os modelos explicativos para o efeito de priming semântico na leitura. Por fim, serão analisadas pesquisas utilizando o paradigma para investigar o reconhecimento de palavras escritas.

No priming semântico há uma relação de significado ou de contexto entre o prime e o alvo. O efeito de priming semântico ocorre quando uma palavra, que é precedida por outra semanticamente relacionada, é processada de forma mais rápida e acurada (Davenport \& Potter, 2005). O contexto semântico (ou seja, o prime) também pode ser representado por figuras ou sentenças.

Considerando os aspectos metodológicos, nos experimentos de priming semântico, apresenta-se o prime (ex. gato), seguido pela apresentação de um estímulo-alvo (ex. cachorro). Mede-se a acurácia e/ou velocidade de resposta à palavra alvo. Manipula-se o contexto semântico, ou seja, algumas vezes o alvo é precedido ou ativado por uma palavra ou sentença semanticamente relacionada e em outras, condições controle, ele é precedido por uma palavra ou sentença não relacionada ou por prime neutro (não lingüístico) (Nievas \& Justicia, 2004). Pode-se também variar ou controlar a relação prime-alvo (categórica ou temática), as características psicolingüísticas do alvo (extensão, freqüência), o intervalo entre a apresentação do prime e o início do alvo (Stimulus Onset Asynchrony SOA).

O priming semântico, assim como outros tipos de priming (ortográfico, fonológico), pode ser investigado na forma disfarçada (masked priming), em que o prime é apresentado entre máscaras (ex.: \#\#\#\# ou \&\&\&\&) e tão rapidamente que o participante não é consciente da sua existência, ou na forma de priming visível, no qual o tempo de apresentação do prime é superior ao limiar de consciência do estímulo.

No priming disfarçado, as máscaras são utilizadas para impedir que a imagem do prime permaneça na retina. Uma palavra apresentada por poucos milissegundos (ms) pode até ser legível, mas, se esta for apresentada em proximidade espacial e temporal a um estímulo visual, como \#\#\#, pode se tornar indistinguível ou até mesmo invisível (Dehaene e cols., 2001).

Utilizam-se, em geral, dois tipos de tarefas experimentais para testar o efeito de priming semântico no processamento de palavras: nomeação ou leitura e decisão lexical. Nas tarefas de nomeação, o participante é instruído a ler em voz alta os alvos apresentados, o mais rápido e precisamente possível. Na decisão lexical, os participantes têm que decidir, o mais rapidamente possível, se o alvo é uma palavra ou uma pseudopalavra, ou seja, estímulo formado por uma combinação de fonemas ou grafemas que não existem no léxico de uma língua. Na tarefa de decisão lexical, independente de ser utilizada em um estudo de priming semântico, o tempo decorrido para aceitar certo estímulo como palavra é inferior ao decorrido em recusar estímulos categorizados como pseudopalavras. A maior latência para as pseudopalavras é considerada indício de que o sujeito fez uma busca, que eventualmente acaba em fracasso, em seu léxico interno (Eysenck \& Keane, 1994), que são as memórias que arquivam entidades lexicais, como as palavras.

Antes de tratar especificamente da interpretação dos experimentos de priming semântico na investigação do processamento de leitura, cabe mencionar brevemente os modelos cognitivos de processamento visual de palavras. Na Neuropsicologia Cognitiva e na Psicologia Cognitiva, a leitura é considerada uma atividade complexa que envolve dois processos fundamentais e interdependentes: o reconhecimento de palavras, foco do presente artigo, e a compreensão do que é reconhecido.

O reconhecimento de palavras impressas ou acesso ao léxico mental, consiste no acesso à palavra na memória permanente, dado um input gráfico, resultando 
na codificação do significado das palavras (Perfetti, 1992). Carmona, González e Gómez (1992) sugerem algumas etapas de acesso ao léxico: 1) contato léxico inicial, ou seja, recepção do input visual e sua transformação em representações fonológicas ou ortográficas; 2) ativação, na qual as palavras que apresentam maior freqüência de uso encontrem-se mais disponíveis ou mais ativadas, resultando em uma maior probabilidade de serem selecionadas; 3) seleção, onde há um progressivo abandono de candidatos léxicos até que o sistema retenha um, que é finalmente a entrada léxica reconhecida pelo sujeito; 4) reconhecimento, propriamente dito, é o momento preciso em que a palavra é identificada.

Dois tipos de processamento cognitivo atuam interativamente no acesso ao léxico mental, os ascendentes (bottom-up) e os processos descendentes (top-down), ou seja, a informação pode fluir em ambas as direções. Nos processos ascendentes, as informações são passadas dos processos de nível mais baixo, tais como a identificação de linhas e ângulos, para os níveis intermediários, tais como a identificação da letra, e daí para o reconhecimento de uma palavra. Nos processos descendentes, o contexto ortográfico e semântico intervém na identificação das palavras. O fenômeno de priming semântico se presta à investigação da influência destes processos descendentes na leitura.

Há basicamente duas abordagens teóricas para explicar a leitura de palavras: os modelos de leitura de Dupla-Rota (abordagem de Processamento da Informação) e os modelos de Processamento Paralelo Distribuído (abordagem Conexionista). De acordo com os modelos de Leitura de Dupla-Rota, o reconhecimento de palavras, em um sistema alfabético de escrita, pode ocorrer por meio de um processo visual direto (rota lexical) ou através de um processo envolvendo mediação fonológica (Coltheart, Curtis, Atkins \& Haller, 1993; Coltheart, Rastle, Perry, Langdon \& Ziegler, 2001; Ellis, 1995; Ellis \& Young, 1988; Hillis \& Caramazza, 1992; Lecours, Tainturier \& Parente, 1997; Patterson, Marshall \& Coltheart, 1985).

A rota fonológica utiliza o processo de conversão grafema-fonema e permite a pronúncia precisa das palavras que possuem correspondência letra-som regular. As representações fonológicas construídas são usadas para obter a informação semântica das palavras (Kay \& Patterson, 1985). Na rota lexical, geralmente utilizada por leitores proficientes, as representações de palavras familiares são armazenadas em um léxico de entrada visual, que permite acesso direto ao significado.
A utilização exclusiva desta rota acarreta muita dificuldade com palavras não-familiares e pseudopalavras.

Alternativamente aos modelos de leitura de DuplaRota, os modelos conexionistas, como o de Processamento Paralelo-Distribuído (PPD), de Seidenberg e McClelland (1989), propõem uma única rota de leitura. As informações ortográfica, fonológica e semântica são representadas em termos de padrões distribuídos de atividade em grupos distintos de unidades de processamento, semelhantes a neurônios. Em cada domínio, as palavras semelhantes são representadas por padrões similares de atividades (Plaut, MacClelland, Seidenberg \& Patterson, 1996).

Conforme Plaut e cols. (1996), a leitura oral requer que pelo padrão ortográfico de uma palavra se gere uma representação fonológica apropriada. Tais transformações são executadas via interações cooperativas e competitivas entre unidades ortográficas, semânticas e fonológicas. Estas interações entre as unidades são governadas por pesos das conexões entre elas, cujos valores dependem da exposição do leitor a palavras escritas, palavras faladas e seus significados (conhecimento prévio).

Nesta abordagem, segundo Plaut e cols. (1996), a leitura de palavras irregulares e de pseudopalavras envolvem processos homogêneos, diferindo dos modelos de Dupla-Rota, que propõem processos distintos. Conflitos entre possíveis pronúncias alternativas de uma série de letras são resolvidos por interações cooperativas e competitivas, com base no conhecimento disponível de palavras e suas pronúncias. Além disso, a representação semântica de uma palavra participa na leitura oral exatamente da mesma maneira que as representações ortográficas e fonológicas.

Conforme exposto, são vários os fatores psicolingüísticos que influenciam o reconhecimento de palavras, como freqüência de uso, número de vizinhos ou competidores que a palavra possui no léxico mental, regularidade da correspondência grafema-fonema, entre outros. Esses fatores deveriam ser considerados no delineamento de experimentos de priming semântico para estudar o processamento de leitura.

É possível supor que a abordagem conexionista de acesso ao léxico explique melhor o efeito de priming semântico na leitura, comparado aos modelos de processamento da informação. Uma destas explicações teóricas para o priming semântico, conforme Brown, Roberts e Besner (2001), é a abordagem de propagação da ativação (Anderson, 2004; Coltheart e cols., 1993). Segundo a mesma, a apresentação de um prime 
(ex. gato) ativa a representação apropriada no nível de letras (as letras correspondentes ao estímulo tornam-se ativadas), que então ativa o nível lexical "Gato" (representações de palavra daquelas letras tornam-se também ativadas). A ativação segue para o nível de representação semântica de Gato e, de forma mais branda, para os associados semânticos (ex. cachorro). Apesar de "Gato" ser o melhor candidato para a representação no nível semântico, ocorre a ativação de outras palavras relacionadas semanticamente, via propagação da ativação (Reimer, Brow \& Lorsbach, 2001).

Neely (1991), ao discutir as teorias que podem explicar o priming semântico, mostrou que quando a assincronia de início dos estímulos (SOA) é pequena (menos do que $300 \mathrm{~ms}$ ), ou seja, quando o prime é apresentado rapidamente (o que implica processos automáticos), as teorias de propagação da ativação (Anderson, 1983) explicam melhor os dados. Essas teorias são baseadas nos pressupostos de que nodos relacionados semanticamente/associativamente são estocados em forma de rede. A ativação do nodo do prime espalha-se na rede para palavras semanticamente relacionadas. Desta forma, reduz o tempo exigido para níveis de ativação daqueles nodos para alcançar o limiar de reconhecimento destas palavras.

Contudo, quando a assincronia de início dos estímulos (SOA) é maior (mais do que $300 \mathrm{~ms}$ ), ou seja, o prime é apresentado por um tempo mais longo, o priming semântico pode ser explicado por processos estratégicos, nos quais estão implicados processos atencionais (Neely, 1991). Nestes casos, teorias de expectativa (expectancy theories) explicariam o efeito de priming, assumindo que os participantes usam o prime para gerar uma série de expectativas que consistem em alvos potenciais relacionados ao prime. Alvos incluídos nesta série esperada são reconhecidos mais rapidamente do que aqueles que não estão na série.

Conforme Brown e Besner (2002), o efeito de priming semântico e sua hipótese explicativa estão relacionados à consciência ou não dos primes (prime visível $\mathrm{X}$ disfarçado) e à consciência ou não da relação semântica entre primes e alvos. Esta última depende da proporção de pares prime-alvo relacionados semanticamente, com relação à proporção de pares não relacionados no experimento. Quando o prime é visível, a proporção de pares semanticamente relacionados é alta e os participantes estão conscientes disso, o processamento do prime deve envolver feedback do nível semântico para o lexical (processos atencionais).
Quando os participantes não são conscientes do prime (ex.: quando ele é apresentado por $34 \mathrm{~ms}$ ), este feedback não ocorre (processos automáticos).

A seguir serão abordados alguns estudos empíricos que utilizaram o paradigma de priming semântico para investigar o acesso a informações semânticas no processamento visual de palavras. Esta revisão pretende demonstrar as formas de aplicação do paradigma de priming semântico na investigação do processamento de leitura, mais especificamente no acesso à informação semântica.

\section{Investigação experimental do processamento de leitura através do paradigma de priming semântico}

Na literatura internacional há um grande número de publicações utilizando o paradigma de priming na investigação do processamento de leitura. Em amostras de universitários, constatou-se a existência de efeitos de priming fonológico, como exemplo, o par "foca - faca" (Carreiras, Ferrand, Grainger \& Perea, 2005; Lukatela, Eaton, Lee \& Turkey, 2001; Lukatela \& Turvey, 1991; Pollatsek, Perea \& Carreiras, 2005) e de priming semântico (ex.: "isca - peixe”) no processamento de leitura (Brown \& Besner, 2002; Brown e cols., 2001; Friedrich, Henik \& Tzelgov, 1991; Nievas \& Justicia, 2004; Reimer e cols., 2001).

Voltando-se para os processos de leitura nos leitores em desenvolvimento (aprendizes), têm sido encontrados efeitos de priming ortográfico e fonológico (Booth, Perfetti \& MacWhinney, 1999; Bowey, Vaughan \& Hansen, 1998), assim como de priming semântico (Assink, Bergen, Teeseling \& Knuijt, 2004; Simpson \& Forster, 1986; Simpson \& Lorsbach, 1983).

Porém, no Brasil, as publicações enfocando os estudos no paradigma de priming, mesmo não restritos ao contexto do processamento de leitura, são escassas (Azevedo, Squella \& Ribeiro-do-Valle, 2001; Busnello, 2007; Carreiro, Haddad Júnior \& Baldo, 2003; DelFava \& Ribeiro-do-Valle, 2004; Macedo, 2003; Oliveira, 2001; Oliveira \& Janczura, 2004; Pompéia \& Bueno, 2006; Pompéia, Paes \& Bueno, 2003; Rosin, 2001, 2004; Squella \& Ribeiro-do-Valle, 2003), conforme pesquisa feita nas bases de dados Scielo e LILACS, com a palavra-chave "priming”.

Ainda no cenário internacional, concentrando-se nos estudos que investigaram o priming semântico em leitores adultos/proficientes, Lukatela e Turvey (1991), utilizando o paradigma de priming com pseudohomófonos, encontraram uma interação entre contexto 
semântico (associação semântica) e fonológico (pseudo-homofonia). Nesta pesquisa os estímulos-alvo eram pseudopalavras que soavam como palavras reais e os primes eram palavras que estavam relacionadas semanticamente às palavras com as quais cada alvo era homofônico (Ex.: table - chare - pseudohomófono de chair). Pseudo-homófonos como "Chare" foram lidos mais rapidamente e com menos erros quando o contexto foi "table" (semanticamente relacionado) do que quando o contexto foi "novel” (controle).

O efeito de priming semântico depende do tipo de processamento realizado no prime. Na pesquisa de Brown e cols. (2001), também com universitários, era apresentada primeiro uma série de letras (ex.: PPPPPP) e, ao mesmo tempo, dois primes: um que o sujeito deveria atentar (ex.: PRETTY) e outro que o sujeito era instruído a ignorar (ex. grow). Em ambas condições, os primes poderiam ser relacionados ou não relacionados semanticamente ao alvo. Para um grupo de participantes a instrução era indicar se o prime que ele deveria atentar continha a letra $\mathrm{P}$, neste caso. Para outro grupo era solicitada a leitura do prime atentado. Após esta série de estímulos, o alvo era apresentado para o participante realizar uma decisão lexical. Os resultados mostraram efeito de priming tanto na condição de prime ignorado quanto atentado, quando solicitada a leitura do prime. Porém, na condição de identificação de letra no prime não houve efeito de priming semântico.

Segundo Brown e cols. (2001), a ausência do efeito de priming semântico na condição de identificação de letra no prime é consistente com a conclusão de que quando o processamento é dirigido para o nível da letra, um bloqueio de ativação é construído entre os níveis de representação lexical e semântico, conforme modelos de propagação da ativação.

Friedrich e cols. (1991) também apontam que a natureza do processamento do prime parece determinante para a ocorrência de efeito de priming semântico para palavras alvo. Ainda em estudos com leitores adultos, efeitos de priming semântico foram encontrados em alguns estudos quando o prime não requeria qualquer tipo de resposta, quando ela era lida em voz alta, quando uma decisão lexical era feita sobre o prime e quando um julgamento sintático (substantivo - não substantivo) ou semântico (agradável/não agradável) era exigido (Henik, Friedrich \& Kellogg, 1983; Smith, Theodor \& Franklin, 1983).

Em relação ao tempo de apresentação do prime, Perea e Gotor (1997) estudaram os efeitos de priming associativo e semântico em universitários em tarefas de decisão lexical e de leitura de palavras, através do paradigma de masked priming (priming disfarçado). Os resultados mostraram fracos, mas significativos, efeitos de priming associativo em ambas tarefas. Comparando três tempos de apresentação do prime (33, 50 e 67 milissegundos), o procedimento utilizando 67 milissegundos mostrou efeitos de priming facilitatório para os pares relacionados semanticamente. Os resultados deste estudo são consistentes com os modelos interativos de reconhecimento de palavras, nos quais a ativação semântica pode influenciar os estágios iniciais de processamento da palavra. Parece que o estudo de efeito de priming semântico é difícil de ser encontrado quando o participante não tem consciência do prime e o SOA é muito breve.

Em se tratando da investigação da leitura de palavras em crianças/adolescentes, há muito poucos estudos utilizando o paradigma de priming semântico (Nievas \& Justicia, 2004; Simpson \& Forster, 1986; Simpson \& Lorsbach, 1983, 1987) e encontram-se menos ainda com relação a crianças com dificuldades de leitura (Assink e cols. 2004; Nation \& Snowling, 1999) ou com outro transtorno de desenvolvimento, como autismo (Hala, Pexman \& Glenwright, 2007). Além dessa escassez de estudos com crianças utilizando o paradigma de priming semântico, os resultados são divergentes.

Sabe-se que crianças com dificuldades de leitura (dislexias de desenvolvimento) têm alterações no acesso ao léxico mental (Wise, Ring \& Olson, 1999), ou seja, nos processos mais básicos de leitura, o que certamente compromete o desenvolvimento da leitura compreensiva. Ainda não está bem estabelecida na literatura a natureza destas dificuldades, dependendo do tipo de dislexia. A maioria dos estudos encontrados (Beitchman \& Young, 1997; Jong, 1998; Mayringer \& Wimmer, 2000; Morais, 1996; Pennington, 1997; Salles, 2005; Salles \& Parente, 2006a, 2006b; Torgesen, Wagner \& Rashotte, 1994; Wagner \& Torgesen, 1987; Wimmer, 1993) versa sobre as dificuldades no processamento fonológico da linguagem como fator subjacente às dificuldades de leitura.

Uma questão a ser melhor investigada é se alterações no acesso a informações semânticas das palavras lidas poderiam estar relacionadas às dificuldades de leitura nas crianças. O paradigma de priming semântico permite a análise on-line do processamento de leitura.

Uma das posições apresentadas na literatura é de que os processos de reconhecimento de palavras de 
crianças mais novas têm mais benefícios do contexto semântico (priming semântico) do que aqueles de crianças mais velhas (Simpson \& Lorsbach, 1983). Este decréscimo de efeito de priming semântico relacionado à idade tem sido demonstrado em tarefas usando decisão lexical e leitura oral de palavras (Nievas \& Justicia, 2004). Neste caso, o contexto semântico seria usado para compensar as dificuldades iniciais no reconhecimento das palavras escritas.

Por outro lado, não aparece o efeito do priming semântico em crianças quando o prime é apresentado muito rapidamente (Nievas \& Justicia, 2004; Simpson \& Forster, 1986). Quanto mais longa é a apresentação do prime, crianças de séries iniciais têm maior efeito de priming do que as crianças de séries superiores (Schwantes, Boesl \& Ritz, 1980).

Simpson e Lorsbach (1983) compararam os efeitos de priming semântico entre crianças de $2^{\mathrm{a}}$ (8 anos), $4^{\mathrm{a}}$ (anos) e $6^{a}$ séries (12 anos) e adultos (25 anos). Os alvos eram apresentados nas condições "com prime relacionado" (ex.: dog-cat), "com prime não relacionado" e "com prime neutro" (ex.: XXX). Os primes apareciam por 2 segundos e o participante deveria ler em voz alta o alvo apenas. Os resultados mostraram efeito de priming semântico, mas o efeito de facilitação dos primes relacionados semanticamente diminuiu com a idade/escolaridade. Utilizando o mesmo método de estudo, Simpson e Lorsbach (1987) encontraram efeito de priming semântico em crianças de $4^{\mathrm{a}}$ e $6^{\mathrm{a}}$ séries, tanto leitoras medianas quanto naquelas com leitura acima da média.

Em outro estudo (Simpson \& Forster, 1986) a magnitude da facilitação contextual no reconhecimento de palavras por crianças decresceu com a idade. Os autores realizaram um experimento de priming semântico com crianças de $2^{\mathrm{a}}$, $4^{\mathrm{a}}$ e $6^{\mathrm{a}}$ séries. As crianças deveriam ler as palavras alvo, que eram precedidas por primes com mais de um significado (ambíguos). Os alvos estavam relacionados a um dos sentidos do prime (ex.: "banco" de sentar ou agência financeira) ou não estavam relacionadas semanticamente ao prime. As crianças eram instruídas a olhar a primeira palavra (prime) apresentada na tela, por 500 milissegundos, mas não lê-la em voz alta. O alvo deveria ser lido em voz alta o mais rapidamente possível. Os resultados mostraram tempos de reação (TRs) mais rápidos para alvos precedidos por primes com relação semântica do que primes não relacionados. Comparações entre séries mostraram que as maiores mudanças quantitativas (considerando TRs) no uso do contexto parecem ocorrer entre $2^{\mathrm{a}}$ e $4^{\mathrm{a}}$ série, com a facilitação de palavras relacionadas decrescendo entre as séries.

Em um segundo experimento também com crianças de $2^{\mathrm{a}}$, $4^{\mathrm{a}}$ e $6^{\mathrm{a}}$ séries, Simpson e Forster (1986) manipularam o intervalo entre a apresentação do prime e do alvo (SOA). Quando os SOAs foram 150 e 300 milissegundos, os tempos de reação na leitura dos alvos diminuíram com a idade. As crianças foram hábeis em utilizar informação suficiente do prime para facilitar o reconhecimento do alvo, mesmo quando o prime foi apresentado durante $150 \mathrm{~ms}$.

No estudo de Nievas e Justicia (2004), conduzido na Espanha e com método semelhante ao de Simpson e Forster (1986), quando a assincronia de início dos estímulos (SOA) era pequena, ou seja, o prime permanecia na tela por apenas 250 ms, não houve efeito de priming semântico (com homógrafos) em crianças de $5^{\mathrm{a}}$ e de $8^{\mathrm{a}}$ séries, mas houve em adultos de $1^{\circ}$ ano e de $3^{\circ}$ ano do Ensino Médio e universitários. Os autores acreditam que esta ausência de efeito de priming semântico com SOAs curtos é devido a déficit no processamento da palavra, relacionado a acesso ao significado e não a processos de decodificação de palavras. Uma estratégia para compensar tal déficit seria aumentar tempo de exposição ao prime, o que conduziria a utilização de processos controlados ou atencionais, que são produzidos quando o SOA é longo. De acordo com a teoria de propagação da ativação, esta falta de efeito de priming semântico quando o prime é apresentado muito rápido deve-se a um déficit na força associativa dos nodos ou talvez um déficit no número de nodos que são conectados ao nodo alvo (Nievas \& Justicia, 2004).

Ainda sobre este resultado também se pode pensar que a menor velocidade de acesso à informação ortográfica e fonológica das palavras em algumas crianças que estão aprendendo a ler pode comprometer o aparecimento do efeito de priming quando o prime é apresentado rapidamente. É importante também considerar que neste estudo, diferente do de Simpson e Forster (1986), a tarefa era de decisão lexical sobre o alvo, ou seja, os alvos variavam entre palavras reais e pseudopalavras. Decisão lexical, ao contrário de leitura de palavras, não é uma tarefa familiar para crianças. Ainda, a amostra do estudo de Simpson e Forster (1986) era formada por crianças americanas de escolas públicas, incluindo de $2^{\mathrm{a}}$ e $4^{\mathrm{a}}$ série, enquanto que a amostra do estudo de Nievas e Justicia (2004) compreendia crianças espanholas apenas de $5^{\mathrm{a}}$ série.

Com relação à comparação dos efeitos de priming semântico entre leitores com e sem dificuldades, 
Assink e cols. (2004) investigaram se haveria alguma evidência para déficit semântico em crianças holandesas que apresentam fracas habilidades de leitura de palavras, através de tarefa de decisão lexical. Os primes apareciam por 500 milissegundos. Os resultados deste estudo mostraram efeitos principais da condição de priming, ou seja, latências para primes relacionados menores do que para primes não relacionados e do que para primes neutros (não lingüístico). Leitores fracos foram significativamente mais lentos do que os participantes dos grupos controle apenas na condição priming neutro. Não houve evidências para um déficit semântico genérico, em função de não ter havido diferenças significativas entre leitores com dificuldade e leitores competentes na condição de priming semântico.

Assink e cols. (2004) explicam os resultados deste estudo em termos de efeitos ortográficos puros. Os primes neutros eram não lingüísticos (não continham nem informação ortográfica, nem semântica). Os leitores fracos foram mais dependentes das relações grafema-fonema (contidas nas condições primes relacionados e não relacionados ao alvo). Quando a informação ortográfica estava disponível, seu desempenho não diferiu significativamente ao dos grupos controles.

Esses dados corroboraram os achados de Vellutino, Scanlon e Spearing (1995) de que o desempenho no reconhecimento de palavras do grupo de leitores fracos pode ser caracterizado como uma deficiência na velocidade de acesso a links grafema-fonema, resultando em lentidão no reconhecimento de palavras. Os efeitos nas condições de priming relacionado e não relacionado refletiram a dependência das crianças em relação a estas relações, e esta dependência torna-se visível apenas na condição de priming neutro.

Nation e Snowling (1999) estudaram crianças com dificuldade de compreensão textual, que apresentavam boas habilidades de decodificação, com o objetivo de investigar a natureza do conhecimento de palavras destas crianças, usando o paradigma de priming semântico. Porém, neste estudo a apresentação das palavras foi na modalidade oral e não-visual. O estudo comparou estas crianças com dificuldade de compreensão textual com leitores normais, emparelhados por habilidade de decodificação, idade cronológica e habilidade não-verbal. Os resultados mostraram que ambos os grupos mostraram efeitos de priming para palavras relacionadas semanticamente pela função (ex.: vassoura-chão). Nos bons leitores apareceu o efeito de priming para alvos relacionados semanticamente pela categoria (ex.: gato-cachorro), independente da força de associação prime-alvo, enquanto que nas crianças com dificuldade apenas encontrou-se efeito de priming nesta condição se a força de associação entre prime-alvo era alta. Os autores concluem que crianças com dificuldades de compreensão textual são menos sensíveis a relações semânticas abstratas do que as sem dificuldade, considerando o processamento da linguagem oral e não escrita.

As diferenças encontradas entre os resultados de Assink e cols. (2004) e os de Nation e Snowling (1999) podem ser devido à forma de seleção dos grupos (diferenças na leitura de palavras X compreensão textual), assim como o fato do primeiro avaliar o processamento visual de palavras enquanto o de Nation e Snowling avaliar o processamento da palavra na modalidade oral.

Estudando o efeito de priming semântico em crianças com autismo, comparado a crianças com desenvolvimento típico, Hala e cols. (2007) utilizaram três tipos de condições experimentais: primes semanticamente relacionados a cada um dos significados de alvos homógrafos (palavras com mesma grafia que representam mais de um significado) (ex.: RIP-TEAR; CRY-TEAR); primes semanticamente relacionados a alvos não homógrafos (ex.: CAT-DOG) e primes não relacionados semanticamente a alvos não homógrafos (ex.: LION-BREAD). Os autores encontraram que, para ambos os grupos de crianças, houve efeito de priming semântico, ou seja, os alvos precedidos por primes semanticamente relacionados foram lidos mais rapidamente. Vale ressaltar que neste estudo os primes permaneciam na tela do computador por $1000 \mathrm{~ms}$ e as crianças deveriam ler os primes. Este método favoreceu a evidência do efeito de facilitação do prime sobre o processamento do alvo.

Cabe salientar que as divergências de resultados nas pesquisas de priming semântico parecem estar relacionadas a questões metodológicas, assim como podem sofrer influência das diferenças inerentes ao sistema de escrita (língua). Em relação ao método, há variações no tipo de tarefa a ser realizada com o alvo (decisão lexical ou leitura); tempo de apresentação do prime (curto ou longo, visível ou não-visível); tipo de prime (ambíguo ou com um único significado); relação semântica entre prime e alvo, se categórica ou temática (por função) (Assink e cols., 2004); tipo de processamento que é feito no prime (ler em voz alta, apenas olhar ou encontrar uma letra no prime); força da associação semântica entre prime e alvo (Nievas \& Justicia, 2004), que depende da forma como as listas de pares associados semânticos são construídas; proporção de pares prime-alvo semanticamente relacionados e de pares não relacionados em cada experimento; número de vizinhos semânticos das palavras; qualidade do alvo, podendo este ser apresentado na 
forma “degradada” (redução do contraste) ou não degradada (Brown \& Besner, 2002), entre outras variações.

Uma outra questão a considerar é que as medidas de tempo de reação (TR) com output oral (leitura em voz alta), especialmente em crianças, podem sofrer uma série de artefatos, como a realização de leitura incompleta do estímulo ou leitura subvocal antes de leitura oral, o que pode gerar erros de medida ou redução do número de respostas válidas.

Com relação às diferenças de resultados entre estudos conduzidos em línguas diferentes, pode-se abordar a influência a própria diferença de idioma e dos fatores psicolingüísticos na seleção dos estímulos, como frequiência de ocorrência na língua, extensão da palavra, concretude, regularidade da correspondência grafema-fonema, entre outros. Além disso, a forma como é feito o emparelhamento semântico prime-alvo pode influenciar nos resultados. Por exemplo, há pesquisas na qual a associação livre ("diga a primeira palavra que lhe vem à mente quando pensa na palavra...”) para construção das listas de pares associados semânticos é feita por adultos, mas a aplicação do experimento é feita em crianças (como em Hala e cols., 2007). Por fim, a forma de seleção dos participantes e as características dos mesmos (idade, escolaridade, proficiência em leitura, língua materna) também podem gerar diferenças nos resultados.

\section{CONSIDERAÇÕES FINAIS}

Nas últimas décadas tem-se utilizado o paradigma de priming nos estudos de reconhecimento de palavras. Com base nesta revisão, é possível perceber que a maioria dos estudos que utilizam o paradigma de priming para investigar o reconhecimento visual de palavras é realizada com leitores proficientes, adultos, especialmente com universitários, e na língua inglesa, francesa ou espanhola. Além disso, a maioria utiliza tarefas de decisão lexical sobre o alvo. Não foram encontrados estudos nacionais utilizando o paradigma de priming semântico para avaliação do processamento de leitura em crianças aprendendo a ler e/ou que apresentam dificuldades de leitura. A investigação dos efeitos de priming semântico em crianças brasileiras faz-se necessária, já que o sistema de escrita do português, apesar de alfabético, apresenta diferenças importantes em relação ao inglês, por exemplo.

O paradigma de priming semântico é, portanto, uma medida indireta do processamento semântico na leitura, que investiga o conhecimento implícito. Permite a investigação do processamento semântico online, ou seja, durante o processamento das palavras lidas e não após o término deste. Com base no exposto, salienta-se a importância dos estudos experimentais para a avaliação de processos cognitivo-lingüísticos, como a leitura. Um delineamento metodológico bem definido, com base em modelos cognitivos de leitura, permite a investigação de aspectos específicos do processamento de acesso ao léxico mental, como o acesso à informação semântica de palavras lidas.

Ainda encontra-se em aberto a discussão sobre os efeitos de priming semântico no processamento de leitura de palavras, especialmente nos leitores em desenvolvimento e naqueles com dificuldades de leitura. O aprofundamento de estudos nesta área tem importantes aplicações para a prevenção, identificação precoce e reabilitação dos problemas de desenvolvimento da leitura, assim como daquelas alterações de leitura adquiridas em função de danos cerebrais (dislexias adquiridas). Portanto, o paradigma de priming semântico, além de recurso metodológico de investigação de processos cognitivos pode ser utilizado no delineamento de programas de intervenção para aprimorar a compreensão de leitura e como estratégia de reabilitação nos casos de transtornos adquiridos de memória (amnésias) e nas demências.

\section{REFERÊNCIAS}

Anderson, J. R. (1983). The architecture of cognition. Cambridge, MA: Harvard University Press.

Anderson, J. R. (2004). Psicologia cognitiva e suas implicações experimentais (D. C. Alencar, Trad.). Rio de Janeiro: LTC.

Assink, E. M. H., Bergen, F. V., Teeseling, H. V., \& Knuijt, P. P. N. A. (2004). Semantic priming effects in normal versus poor readers. The Journal of Genetic Psychology, 165(1), 67-79.

Azevedo, E. L., Squella, S. A. F., \& Ribeiro-do-Valle, L. E. (2001). The early facilitatory effect of a peripheral spatially noninformative prime stimulus depends on target stimulus features. Brazilian Journal of Medical and Biological Research, 34, 803-813.

Beitchman, J. H., \& Young, A. R. (1997). Learning disorders with a special emphasis on reading disorders: A review of the past 10 years. Journal of American Academy of Child and Adolescent Psychiatry, 36(8), 1020-1032.

Booth, J. R., Perfetti, C. A., \& MacWhinney, B. (1999). Quick, automatic and general activation of orthographic and phonological representations in young readers. Developmental psychology, 35(1), 3-19.

Bowey, J. A., Vaughan, L., \& Hansen, J. (1998). Beginning readers' use of orthographic analogies in word reading. Journal of Experimental Child Psychology, 68, 108-133.

Brown, M., \& Besner, D. (2002). Semantic priming: On the role of awareness in visual word recognition in absence of an expectancy, Consciousness and Cognition, 11, 402-422.

Brown, M. S., Roberts, M. A., \& Besner, D. (2001). Semantic processing in visual word recognition: Activation blocking and 
domain specificity. Psychonomic Bulletin e Review, 8(4), 778784.

Busnello, R. H. (2007). Efeito de priming subliminar no acesso ao léxico. Dissertação de mestrado não-publicada, Pontifícia Universidade Católica do Rio Grande do Sul, Porto Alegre.

Carmona, M. B., González, J. M. I., \& Gómez, A. R. (1992). Psicología del lenguage: Investigación y teoría. Madrid: Editorial Trotta.

Carreiro, L. R. R., Haddad Júnior, H., \& Baldo, M. V. C. (2003). The modulation of simple reaction time by the spatial probability of a visual stimulus [Resumo]. Brazilian Journal of Medical and Biological Research, 36, 907-911. Retirado em 8 de maio de 2007, da base de dados SciELO.

Carreiras, M., Ferrand, L., Grainger, J., \& Perea, M. (2005). Sequencial effects of phonological priming in visual word recognition. Psychological Science, 16(8), 585-589.

Coltheart, M., Curtis, B., Atkins, P., \& Haller, M. (1993). Models of reading aloud: Dual-rote and parallel-distributed-processing approaches. Psychological Review, 100(4), 589-608.

Coltheart, M., Rastle, K., Perry, C., Langdon, R., \& Ziegler, T. (2001). DRC: Dual-route cascaded model of visual word recognition and reading aloud. Psychological Review, 108(1), 204-256.

Davenport, J. L., \& Potter, M. C. (2005). The locus of semantic priming in RSVP target search. Memory \& Cognition, 33(2), 241-248.

Dehaene, S., Naccache, L., Cohen, L., Le Bihan, D., Mangin, J. F., Poline, J. B., \& Rivière, D. (2001). Cerebral mechanisms of word masking and unconscious repetition priming. Nature, 4(7), 752-758.

Del-Fava, F., \& Ribeiro-do-Valle, L. E. (2004). Relative contribution of expectancy and immediate arousal to the facilitatory effect of an auditory accessory stimulus. Brazilian Journal of Medical and Biological Research, 37, 1161-1174.

Ellis, A. W. (1995). Leitura, escrita e dislexia: Uma análise cognitiva (D. Batista, Trad.). Porto Alegre: Artes Médicas.

Ellis, A. W., \& Young, A. W. (1988). Human cognitive neuropsychology. London: Lawrence Erlbaum Associates.

Eysenc, M. W., \& Keane, M. T. (1994). Psicologia cognitiva: Um manual introdutório (W. Gesser \& M. H. F. Gesser, Trads.). Porto Alegre: Artes Médicas.

Forster, K. I. (1999). The microgenesis of priming effects in lexical access. Brain and Language, 68, 5-15.

Friedrich, F. J., Henik, A., \& Tzelgov, J. (1991). Automatic processes in lexical access and spreading activation. Journal of Experimental Psychology: Human Perception and Performance, 17(3), 792-806.

Hala, S., Pexman, P. M., \& Glenwright, M. (2007). Priming the meaning of homographs in typically developing children and children with autism. Journal of Autism Developmental Disorder, 37, 329-340.

Henik, A., Friedrich, F. J., \& Kellogg, W. A. (1983). The dependence of semantic relatedness effects upon prime processing. Memory \& Cognition, 11, 366-373.

Hillis, A. E., \& Caramazza, A. (1992). The reading process and its disorders. Em D. I. Margolin (Org.), Cognitive neuropsychology in clinical practice (p. 229-261). New York/Oxford: Oxford University Press.
Jong, P. F. (1998). Working memory deficits of reading disabled children. Journal of Experimental Child Psychology, 70, 75-96.

Kay, J., \& Patterson, K. E. (1985). Routes to meaning in surface dyslexia. Em K. E. Patterson, J. C. Marshall \& M. Coltheart (Orgs.), Surface dyslexia: Neuropsychological and cognitive studies of phonological reading (p. 79-103). London, UK: Lawrence Erlbaum Associates.

Lecours, A. R., Tainturier, M. J., \& Parente, M. A. M. P. (1997). Teoria dos códigos alfabéticos. Em A. R. Lecours \& M. A. M. P. Parente (Orgs.), Dislexia: Implicações do sistema de escrita do português (p. 57-84). Porto Alegre: Artes Médicas.

Lukatela, G., Eaton, T., Lee, C., \& Turkey, M. T. (2001). Does visual word identification involve a sub phonemic level? Cognition, 78, B41-B52.

Lukatela, G., \& Turvey, M. T. (1991). Phonological access of the lexicon: Evidence from associative priming with pseudohomophones. Journal of Experimental Psychology: Human Perception and Performance, 17(4), 951-966.

Macedo, C. M. R. A. (2003). Efeitos do letramento tardio sobre a organização do conhecimento semântico. Tese de doutorado não-publicada, Universidade Federal de Santa Catarina, Florianópolis.

Mayringer, H., \& Wimmer, H. (2000). Pseudoname learning by german-speaking children with dyslexia: Evidence for a phonological learning deficit. Journal of Experimental Child Psychology, 75, 116-133.

Morais, J. (1996). A arte de ler. São Paulo: Editora da UNESP.

Nation, K., \& Snowling, M. J. (1999). Developmental differences in sensitivity to semantic relations among good and poor comprehenders: Evidence from semantic priming. Cognition, 70, B1-B13.

Neely, J. H. (1991). Semantic priming effects in visual word recognition: A selective review of current findings and theories. Em D. Besner \& G. W. Humphreys (Orgs.), Basic processes in reading, visual word recognition (p. 264-336). New Jersey: Lawrence Erlbaum Associates.

Nievas, F., \& Justicia, F. (2004). A cross-sectional study about meaning access processes for homographs. Cognitive Development, 19, 95-109.

Oliveira, N. R. (2001). A influência da carga afetiva e do tipo de teste na memória para palavras. Dissertação de mestrado nãopublicada, Universidade Nacional de Brasília.

Oliveira, N. R., \& Janczura, G. A. (2004). Memória para palavras em função da carga afetiva e do tipo de teste. Psico, 35(2), 141-149.

Patterson, K. E., Marshall, J. C., \& Coltheart, M. (1985). Surface dyslexia: Neuropsychological and cognitive studies of phonological reading. London, UK: Lawrence Erlbaum Associates.

Pennington, B. F. (1997). Diagnóstico de distúrbios de aprendizagem: Um referencial neuropsicológico (S. P. Netto, R. S. L. Guzzo, S. M. Wechsler, F. S. Wechsler, E. M. P. Yoshida, G. P. Witter, E. M. S. Rosado e L. H. P. Trombeta, Trads.). São Paulo: Pioneira.

Perea, M., \& Gotor, A. (1997). Associative and semantic priming effects occur at very short stimulus-onset asynchronies in lexical decision and naming. Cognition, 62, 223-240.

Perfetti, C. A. (1992). A capacidade para a leitura. Em R. Sternberg (Org.), As capacidades intelectuais humanas: Uma 
abordagem em processamento de informações (D. Batista, Trad.) (p. 72-96). Porto Alegre: Artes Médicas.

Plaut, D. C., McClelland, J. L., Seidenberg, M. S., \& Patterson, K. (1996). Understanding normal and impaired word reading: Computational principles in quasi-regular domains. Psychological Review, 103(1), 56-115.

Pollatsek, A., Perea, M., \& Carreiras, M. (2005). Does conal prime canal more than cinal? Masked phonological priming effects in Spanish with the lexical decision task. Memory and Cognition, 33(3), 557- 565.

Pompéia, S., \& Bueno, O. F. A. (2006). Um paradigma para diferenciar o uso de memória implícita e explícita. Psicologia: Reflexão e Crítica, 19(1), 83-90.

Pompéia, S., Paes, A. T., \& Bueno, O. F. A. (2003). Teste de complementação de letras: Estímulos para uso no Brasil. Psicologia: Teoria e Pesquisa, 19, 65-73.

Reimer, J. F., Brow, J. S., \& Lorsbach, T. C. (2001). Orthographically mediated inhibition effects: Evidence of activational feedback during visual word recognition. Psychonomic Bulletin e Review, 8(1), 102-110.

Rosin, F. M (2001). Algumas contribuições experimentais ao estudo do efeito de "priming" negativo em tarefas de atenção seletiva. Tese de doutorado não-publicada, Universidade de São Paulo, Ribeirão Preto.

Rosin, F. M. (2004). Assessing negative priming by attended distractors in a paper-and-pencil task. Brazilian Journal of Medical and Biological Research, 37, 1.131-1.153.

Salles, J. F. (2005). Habilidades e dificuldades de leitura e escrita em crianças de $2^{a}$ série: Abordagem neuropsicológica cogniti$v a$. Tese de doutorado não-publicada, Curso de Pós-Graduação em Psicologia do Desenvolvimento, Universidade Federal do Rio Grande do Sul, Porto Alegre.

Salles, J. F., \& Parente, M. A. M. P. (2006a). Heterogeneidade nas estratégias de leitura/escrita em crianças com dificuldades de leitura e escrita. Psico, 37(1), 83-90.

Salles, J. F., \& Parente, M. A. M. P. (2006b). Funções neuropsicológicas em crianças com dificuldades de leitura e escrita. Psicologia: Teoria e Pesquisa, 22(2), 153-162.

Schwantes, F. M., Boesl, S. L., \& Ritz, E. G. (1980). Children’s use of context in word recognition: A psycholinguistic guessing game. Child Development, 51, 730-736.
Seidenberg, M. S., \& McClelland, J. L. (1989). A distributed, developmental model of word recognition and naming. Psychological Review, 97, 477-452.

Simpson, G. B., \& Forster, M. R. (1986). Lexical ambiguity and children's recognition. Developmental Psychology, 22(2), 147154.

Simpson, G. B., \& Lorsbach, T. C. (1983). The development of automatic and conscious components of contextual facilitation. Child Development, 54, 760-772.

Simpson, G. B., \& Lorsbach, T. C. (1987). Automatic and conscious context effects in average and advanced readers. Journal of Research in Reading, 10(2), 102-112.

Smith, M. C., Theodor, L., \& Franklin, P. E. (1983). The relationship between contextual facilitation and depth of processing. Journal of Experimental Psychology: Learning, Memory \& Cognition, 9, 697-712.

Squella, S. A. F., \& Ribeiro-do-Valle, L. E. (2003). Priming effects of a peripheral visual stimulus in simple and go/no-go tasks. Brazilian Journal of Medical and Biological Research, 36, 247-261.

Squire, L. R., \& Kandel, E. R. (2003). Memória: Da mente às moléculas (C. Dalmaz e J. A. Quillfeldt, Trad.). Porto Alegre: Artmed.

Torgesen, J. K., Wagner, R. K., \& Rashotte, C. A. (1994). Longitudinal studies of phonological processing and reading. Journal of Learning Disabilities, 27(5), 276-286.

Vellutino, F. R., Scanlon, D. M., \& Spearing, D. (1995). Semantic and phonological coding in poor and normal readers. Journal of Experimental Child Psychology, 59, 76-123.

Wagner, R. K., \& Torgesen, J. K. (1987). The nature of phonological processing and its causal role in the acquisition of reading skills. Psychological Bulletin, 101(2), 192-212.

Wimmer, H. (1993). Characteristics of developmental dyslexia in a regular writing system. Applied Psycholinguistics, 14, 1-33.

Wise, B. W., Ring, J., \& Olson, R. K. (1999). Training phonological awareness with and without explicit attention to articulation. Journal of Experimental Child Psychology, 72, 271-304.

Recebido: 16/09/2006 Revisado: 31/05/2007 Aceito: 09/06/2007

Nota:

${ }^{1}$ Manuscrito elaborado durante a participação da primeira autora no Programa de Apoio a Projetos Institucionais com a Participação de Recém-Doutores - Prodoc - Capes, no PPG em Psicologia da PUCRS.

\section{Sobre as autoras:}

Jerusa Fumagalli de Salles: Doutora em Psicologia do Desenvolvimento - UFRGS, Prof a Adjunta do Instituto de Psicologia e do Programa de Pós-Graduação em Psicologia da UFRGS.

Graciela Inchausti de Jou: Doutora em Psicologia do Desenvolvimento - UFRGS, Pós-doutoranda do Programa de Pós-Graduação em Psicologia da UFRGS.

Lílian Milnitsky Stein: Doutora em Psicologia Cognitiva, Docente da Faculdade de Psicologia e do Programa de Pós-Graduação em Psicologia da PUCRS.

Endereço para correspondência: Instituto de Psicologia - UFRGS - Rua Ramiro Barcelos, 2600 - Bairro Santa Cecília - Porto AlegreRS - Fone (51) 3308-5111 - Endereço eletrônico: jerusafs@yahoo.com.br 